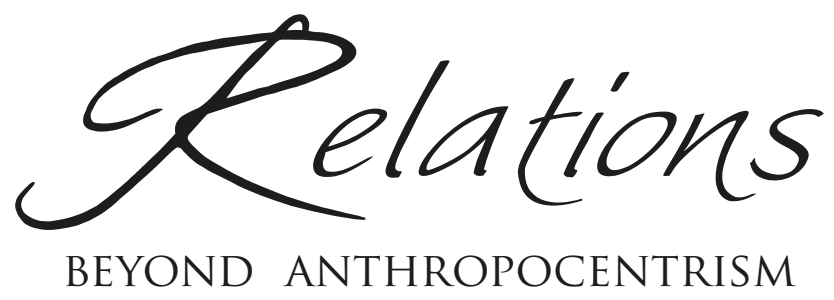

\author{
$9.1-2$ \\ NOVEMBER 2021 \\ Animals: Freedom, Justice, Welfare, \\ Moral Status, and Conflict Cases \\ Edited by Francesco Allegri
}

\author{
STUDIES \\ AND RESEARCH CONTRIBUTIONS
}

Pigs vs. Boars: The Ethics of Assisting Domesticated and Wild Animals

Beka Jalagania

The Chincoteague Ponies and What It Means To Be Free

Alexis Flower

Beyond the Fairy Tale of The Shape of Water: Reimagining the Creature

Viktorija Lankauskaite

Max Scheler e la possibilità di una nuova forma di antispecismo

Enrico R.A. Calogero Giannetto

Distributive Justice and Animal Welfare

Paola Morreale

Unitarianism or Hierarchical Approach for Moral Status?

A Very Subtle Difference

Francesco Allegri 


\section{Comments, Debates, Reports AND INTERVIEWS}

Animals and Justice: The Unfinished Journey

Paola Fossati

Author Guidelines 


\title{
Max Scheler e la possibilità di una nuova forma di antispecismo
}

\author{
Max Scheler and the Possibility of a New Form \\ of Antispeciesism
}

\section{Enrico R.A. Calogero Giannetto}

Università degli Studi di Bergamo

enrico.giannetto@unibg.it

DOI: https://dx.doi.org/10.7358/rela-2021-0102-gian

\begin{abstract}
This article presents the ethical thought of Max Scheler, beyond its anthropocentric specificity, as a possible basis for the philosophical elaboration of an anti-speciesist ethical phenomenology, of Christian origin, which in turn presupposes for the self-understanding of our human existence a vegan and anti-speciesist ethical praxis, as a concrete form of active love as care for every life.
\end{abstract}

Keywords: animals; antispeciesism; christianity; ethics; history of ethics; love; Max Scheler; ontology; phenomenology; veganism.

\section{INTRODUZIONE}

La questione dell'etica animale, come è noto, fra XX e XXI secolo, ha attraversato varie fasi di elaborazione. Nata all'interno di una prospettiva di etica applicata di carattere utilitaristico (Singer 1975) e di una prospettiva dei diritti degli animali (Regan and Singer 1976; Regan 1983; Regan and Linzey 1988), dopo la pubblicazione postuma del libro L'animal que donc je suis di Jacques Derrida (1930-2004) si è trasformata in una problematica di filosofia teoretica: Derrida ha operato una decostruzione delle filosofie che relegavano l'animalità in uno stato di minorità e l'ha associata ad altre decostruzioni importanti come quella del maschilismo e della razionalità umana, nella critica di ciò che ha chiamato "carnofal-lo-go-centrismo" (Derrida 2006). La decostruzione teoretica ha così assunto una connotazione etica (Resta 2003), ma non di un'etica teoretica positiva; piuttosto di una prassi, nell'impossibilità di una teoresi. Dopo, si è compreso che è possibile fornire differenti fondamenti all'antispe- 
cismo, e soprattutto si è cercata una fondazione ontologica o biologica, risalendo anche a filosofie precedenti: si è seguito un percorso in parte invertito rispetto alla storia dell'etica generale, in cui si era passati da una fondazione religiosa e poi metafisica e ontologica a un'autonomia totale dell'etica in Socrate, cui Platone, subito dopo, cercò di ridare una fondazione ontologica e metafisica (Calogero 1932). Un pensiero interessante su cui poter elaborare una prospettiva antispecista vegana è quello della fenomenologia etica di Max Scheler (1874-1928), nonostante il suo antropocentrismo.

\section{LA FENOMENOlOgia ETICA Di Max SCHELER}

Max Scheler è un filosofo ancora oggi quasi del tutto frainteso, trascurato e sottovalutato. Molto spesso si presenta la fenomenologia di Scheler solo come un approfondimento di quella di Edmund Husserl (1859-1938): Scheler si sarebbe occupato, fra tutti gli atti intenzionali umani, degli atti intenzionali etici, cioè di quelli volti a tenere un comportamento etico. Sua specificità sarebbe stata quella di legare gli atti intenzionali etici non all'attività di una razionalità pratica kantiana che governerebbe la volontà che si esplica nell'azione, né all'attività di una razionalità teoretica husserliana che, in quanto disinteressata, fonderebbe direttamente e intellettualmente anche la razionalità pratica etica; ma piuttosto all'attività intenzionale della dimensione affettivo-emotiva della personalità umana: l'etica non si fonda sulla razionalità pratica o teoretica, ma sulla "sensibilità", sul "cuore", in un richiamo a Blaise Pascal. Già questa svolta verso una "fenomenologia trascendentale etica del cuore" (Scheler 1916), rispetto all'etica razionalista dominante, sarebbe importante e poco riconosciuta, ma certamente non è tutto.

Scheler (Scheler 1917, 1921), analizzando la filosofia teoretica platonica, mostra che la prospettiva teoretica non è assunta a priori come disinteressata, ma è piuttosto la conseguenza di un'attività etica che si qualifica come disinteressata in quanto volta a una contemplazione distaccata, a distanza, delle cose: è legata cioè a una vita contemplativa (bios theoretikos) che è considerata la massima pratica etica perché, nella teologia e nella religione popolare greca astrale, è attribuita alle stelle-dèi che stanno a guardare, a distanza, con distacco, le vicende del mondo ed è questo l'atteggiamento che permette di essere felici. La filosofia era cioè legata a una pratica di vita, coinvolgeva l'essere umano nella sua interezza, nella sua esistenza, costituiva un modo di vivere etico, come la sapienza antica richiedeva: il raggiungimento della verità richiedeva una

Relations - 9.1-2 - November 2021

https://www.ledonline.it/Relations/ - Online ISSN 2280-9643 - Print ISSN 2283-3196 
vita etica, che sola poteva assicurare una considerazione delle cose stesse, non finalizzata a vantaggi personali o umani.

Questa consapevolezza porta Scheler a ribaltare la prospettiva della filosofia teoretica husserliana e dominante in generale la filosofia: l'etica, come disciplina che si occupa degli atti intenzionali etici, come parte della filosofia pratica, costituisce la "filosofia prima" su cui si deve fondare la filosofia teoretica e non viceversa; e, ancora di più, l'etica come disciplina richiede una vita etica. Si possono comprendere gli atti intenzionali etici solo in una vita etica. L'intenzionalità etica non riguarda più solo la coscienza come l'intenzionalità husserliana, ma indica un coinvolgimento di tutto l'essere umano (cosa su cui lo seguirà Heidegger con il suo essere-nel-mondo, pur neutralizzando l'aspetto etico) (Heidegger 1927). Si potrebbe dire che c'è un'immanenza del mondo e degli altri nel cuore dell'essere umano, un'immanenza nell'amore come atto.

Tuttavia, nella storia e nella varietà delle etnie, delle culture e delle religioni, si danno differenti ethos, cioè differenti prassi di vita, e differenti etiche; e si può così comprendere la varietà delle filosofie come derivata da una varietà di ethos.

Scheler non condivide l'ethos greco e antico della vita contemplativa: questo implica il distacco dalle cose e dagli altri per preservare una felicità individuale egoistica. Scheler trova nel cristianesimo originario un'altra prassi etica, che considera come attività etica massima non la contemplazione intellettuale, ma piuttosto una vita activa guidata dal cuore e che si compie in un amore quale cura attiva nei confronti delle cose e degli altri. Questa prassi etica porta anche a un'altra forma di comprensione non meramente intellettuale: si comprendono effettivamente le cose e gli altri quando ci si avvicina, eliminando ogni distacco e ogni distanza, nell'amore.

Scheler si rende conto che la prassi etica cristiana non è mai stata elaborata in una filosofia corrispondente: il cristianesimo ha piuttosto assunto come propria dottrina la filosofia teoretica greca, platonica e aristotelica, con lievi modificazioni soltanto. Per Scheler, le intuizioni di Agostino sono rimaste vincolate a una filosofia neo-platonica e la prospettiva di Pascal sulle "ragioni del cuore" non è stata ulteriormente sviluppata (Scheler 1914-1916, 1915): "l'uomo, prima di essere un ens cogitans o un ens volens, è un ens amans".

Scheler opera così un secondo ribaltamento nel ribaltamento, che sta nel rovesciare il rapporto gerarchico fra intelletto e ragione, da una parte, e "sensibilità" e "cuore" dall'altra. Se è il "cuore", cioè la sfera affettivo-emotiva, alla base della vita etica e dell'etica, lo sarà anche alla base della filosofia: si tratterà di sviluppare una "fenomenologia etica", in 
cui gli atti teoretici avranno la loro base negli atti pratici etici, radicati nel cuore. Se la comprensione si realizza nell'amore vissuto, la filosofia come teoria, in quanto trasposizione linguistico-razionale di tale comprensione, resterà, appunto, sempre su un meta-livello rispetto al processo dell'effettiva comprensione delle cose con il cuore, che avverrà sul piano pratico dell'azione etica d'amore (Scheler 1923).

Scheler condivide così con la psicoanalisi la priorità della dimensione affettivo-emotiva su quella intellettuale e la determinazione delle teorie quali razionalizzazioni superficiali di una sfera più profonda, che però non è sede solo di istinti egoistici di piacere o di loro rimozioni patologiche, ma piuttosto anche di "sentimenti" consapevoli, eticamente orientati in modo tale da permetterci un accesso alle cose e agli altri, "coinvolto" e "disinteressato" solo nel senso di essere privo di interessi egoistici negativi. Scheler condivide altresì con le scienze sperimentali la priorità della dimensione pratica su quella teorica e una gnoseologia (una teoria della conoscenza) basata sull'azione e non sul pensiero puro: non però di una pratica tecnica, ma di una pratica etica.

Scheler reinterpreta così la metodologia gnoseologica della fenomenologia di Husserl nei termini di una "metodologia etica", cioè di un discorso che permetta di trovare la "retta via" in senso etico, perché è questa che ci consentirà di accedere e tornare alle cose stesse. L'intuizione categoriale-eidetica, che ci permette di comprendere l' "essenza" delle cose, i fenomeni nella loro "purezza", non è più considerata come un'intuizione intellettuale, ma piuttosto come un'intuizione "sentimentale", un'intuizione "del cuore" che si ha nell'azione etica d'amore, un'intuizione etica: si tratta di una "categoria del cuore" (non dell'intelletto) che impronta la nostra comprensione delle cose. Questa intuizione "del cuore" permette di cogliere nelle cose la dimensione del "valore", cioè quella dimensione di tutte le cose, di tutti i viventi e di tutti gli umani, che richiede il nostro rispetto e la nostra cura ugualmente come nei riguardi di noi stessi.

Qual è l' "essenza" delle cose svelata da questa intuizione etica? Che cosa sono i fenomeni nella loro "purezza”? Queste risposte risultano più chiare dalla reinterpretazione scheleriana della "riduzione fenomenologica" in senso etico. Essa si effettua attraverso tre tipologie di azioni etiche: l'auto-controllo delle pulsioni, l'umiliazione dell'io e l'amore. Non si tratta affatto, cioè, di deprivare le cose dalla loro realtà e dalla loro singolarità per enucleare un eidos ideale e universale come in Husserl, ma piuttosto si tratta, prima di tutto, di depurare le cose, per come si manifestano a noi quali fenomeni, da tutte le connotazioni soggettive e antropocentriche con cui noi li ricopriamo: ci sono connotazioni che derivano 
dalle nostre pulsioni istintive egoistiche, da cui ci libera l'auto-controllo; ci sono connotazioni ulteriori che derivano dal fatto che cogliamo le cose da una prospettiva che è di fatto, inevitabilmente, la nostra realtà individuale-egoica vitale e umana nella nostra storia esistenziale individuale e collettiva, da cui ci libera l'umiliazione dell'io. E, liberati i fenomeni da queste connotazioni soggettive, è l'amore che ci permette positivamente di cogliere le prospettive di tutti gli altri e, al di là della relatività e della parzialità di tutte le connotazioni soggettive e umane, di aprirci così alle cose stesse nella loro intrinsecità, di partecipare al loro essere nell'unione amorosa con esse: l'amore ci permette di cogliere anche gli altri nella loro totalità (al di là delle differenze di etnia, di genere, di cultura e di specie), al di là delle loro pulsioni e delle loro azioni egocentriche e antropocentriche o biocentriche negative, come possibili soggetti etici d'amore. Rapportarsi a tutte le alterità come possibili soggetti d'amore significa comprenderle concretamente nella loro reale "idealità" e nella loro singolare "universalità" etiche, e mai intellettuali, frutto di astrazioni.

Scheler supera così il soggettivismo della fenomenologia husserliana: la realtà non è costituita dall'attività noetica del soggetto umano, ma anzi è necessario depurare $\mathrm{i}$ fenomeni dalle connotazioni soggettive umane per accedere alle realtà che si dona solo nell'azione etica al soggetto e però mai esaustivamente.

Scheler cerca di superare precedenti concezioni dell'amore e dell'etica, ma riuscendoci solo parzialmente e cadendo in varie incoerenze. Scheler vuole fondare un'etica non meramente formale e vuota come quella kantiana, ma che abbia dei contenuti effettivi e materiali, pur restando trascendentale e apriori; vuole spezzare le due identità, da una parte, fra razionale, formale e apriori, dall'altra, fra sensibile, materiale e aposteriori: vuole fondare un'etica trascendentale (apriori), della sensibilità e materiale dei valori. I valori si mostrerebbero all'intuizione eidetica sentimentale forme a priori oggettive delle cose. Anziché fermarsi alla considerazione del "valore" etico intrinseco inestimabile (cioè non traducibile in alcun economico valore d'uso o di scambio) di ogni singolo essere vivente o di ogni singola cosa esistente riconoscibile dall'intuizione etica, Scheler pensa ad un ordine gerarchico, dato a priori, di varie tipologie di valori che vanno dal piacevole all'utile, dall'utile al vitale, dal vitale allo spirituale, e dallo spirituale al sacro come il più elevato. L'atto etico è quello che preferisce il valore più alto, e lo stesso amore è definito come un movimento verso il valore più alto. I valori sono distinti dalle cose-beni che ne sono i concreti portatori, perché esisterebbero in sé a prescindere dai beni concreti mutevoli come un mondo eterno dall'ordine immutabile: l'esempio potrebbe essere la distinzione fra un amico 
concreto empirico che si può perdere per tanti motivi, mentre il valore dell'amicizia resta immutabile e valutabile apriori rispetto agli amici concreti che per noi l'incarnano. Il "mondo dei valori" di Scheler è un nuovo "mondo delle idee" platonico.

Da questo punto di vista, è chiaro che i valori così intesi costituiscono delle astrazioni che conducono a una idealità e a una universalità intellettuali nel cuore dell'etica del sentimento: queste astrazioni intellettuali ricadono ancora in un razionalismo etico soggiacente il sentimento e sono strumentali a una fondazione aprioristica, trascendentale dell'etica. Ma c'è di più: in questa teoria, seppure l'amore è il "sommo bene", è ridefinito come ciò che comporta un movimento, una trasformazione verso valori più alti; e, se questi sono ordinati in una gerarchia fallace come quella posta da Scheler (un ordo amoris che considera i valori del sacro come i più alti), si hanno gravi contraddizioni in cui Scheler medesimo ricade. Così, l'amore potrebbe comportare - a mio parere inaccettabilmente - la pena di morte, l'uccisione in guerra, perché il valore stesso della vita è gerarchicamente inferiore, nell'ordine di Scheler, al valore spirituale giuridico-statale o al valore sacro-religioso per cui si possono effettuare dei sacrifici di vite.

D'altra parte, Scheler aveva introdotto quest'ordine gerarchico dei valori per rifondare l'ordo amoris di Agostino, che si basava su un ordine gerarchico dei beni, che portava a sua volta a gravi incongruenze (Scheler 1914-1916).

Per Agostino, come spiega bene Hanna Arendt (1906-1975) nella sua tesi di dottorato del 1929 (Arendt 1929), l'esistenza umana è contraddistinta platonicamente da un eros che ha come fine il raggiungimento della felicità individuale nell'ottica greca di un'etica eudemonistica: l'eros platonico era un movimento ascendente dai beni particolari del bel corpo e della bella anima all'idea universale ed eterna del bene. Agostino sostituisce all'idea platonica del bene il "sommo bene" che è Dio, e alla contemplazione dell'idea di bene la contemplazione di Dio, che sola ci può rendere felici: l'eros rivolto verso i beni terreni transeunti è terreno, negativo e viene indicato come cupiditas, e l'eros rivolto verso il sommo bene divino eterno è celeste, positivo e viene indicato come caritas. Per quanto Agostino accetti lo specifico cristiano "amore del prossimo", questa forma d'amore ha difficoltà ad essere inserita in questa sua prospettiva platonica, perché il prossimo risulterebbe un bene terreno, dal quale bisogna distogliere l'eros: così, l'amore del prossimo tende ad essere ridotto al desiderio che anche gli altri raggiungano la propria felicità individuale nell'amore di Dio. Secondo Agostino, le cose-beni del mondo sono per essere utilizzati da noi (uti), mentre Dio come sommo bene è per essere 
posseduto e goduto da noi (frui): in ogni caso, la prospettiva dell'etica eudemonistica greca e platonica volta al raggiungimento della propria felicità individuale è fatta propria da Agostino, per cui tutti i rapporti con le cose e con Dio sono comunque strumentali alla propria felicità. Quest'etica agostiniana dei beni non coglie quindi l'essenza dell'amore cristiano quale agàpe, che si distingue dalla pagana "cura di sé" volta alla propria felicità individuale. L'agàpe è "cura attiva e concreta degli altri", volta alla felicità di tutti gli altri, quale dono della propria vita agli altri, anche se questo dono può implicare per sé sofferenza e morte. Nella prospettiva originaria cristiana, essendo Dio l'Amore (I Giov. 4.8 e 4.16), non solo l'amore è un'attività indice di pienezza di realtà e non un'attività indicante una mancanza, ma non ci può essere amore di Dio senza l'amore del prossimo, perché l'amore di Dio non è amore di un oggetto ad esclusione di altri, ma piuttosto è amore dell'Amore di tutto e di tutti, che si dà solo nell'amare gli altri in quanto tali.

La sostituzione da parte di Scheler dell'ordine gerarchico agostiniano dei beni con un ordine gerarchico dei valori gli permette di superare il problema agostiniano dell'amore del prossimo: tutto può essere amato. Tuttavia, come già detto, in Scheler c'è una gerarchia dell'amore subordinata a un ordine di valori: il problema platonico-agostiniano si riproduce su un altro piano in cui alla gerarchia dei beni si sostituisce una gerarchia dei valori. Il ribaltamento cristiano della prospettiva platonica sull'amore consiste nel fatto che l'amore non è il movimento ascendente verso il sommo Bene, ma l'Amore è lo stesso Bene: è il bene in sé ed è volto alla felicità altrui nella loro totalità. Scheler comprende questo ribaltamento parzialmente: definisce l'amore come il movimento ascendente verso il "sommo valore", e, sebbene il fine dell'azione non è la propria felicità individuale, il sommo valore non coincide con lo stesso Amore: il sommo valore può essere quello collettivo, a cui si può sacrificare la vita di un individuo o di molti, come nelle guerre. Scheler comprende che l'amore cristiano è amore personale quale amore nei confronti degli altri come soggetti, ma lo qualifica come puramente spirituale e acosmico confondendolo con l'amore ideale platonico: l'amore cristiano, l'agàpe, come Dio, si fa carne, non si limita a un piano spirituale.

$\mathrm{E}$ in Essenza e forme della simpatia (Scheler 1913, 1923) che Scheler sviluppa una fenomenologia della sfera affettivo-emotiva rilevante per l'etica, distinguendo gerarchicamente le varie forme di sentimento verso gli altri, quali unipatia, empatia, simpatia/compassione, filantropia e amore personale: solo la forma più alta del sentire, l'amore può fondare l'etica.

Nel tentativo di distinguere antropocentricamente in maniera netta le caratteristiche umane da quelle degli altri animali, Scheler pone un'oppo-

Relations - 9.1-2 - November 2021

https://www.ledonline.it/Relations/ - Online ISSN 2280-9643 - Print ISSN 2283-3196 
sizione assoluta fra vita e spirito (Scheler 1928), che resta un presupposto teoretico-metafisico puro rispetto alla sua fenomenologia etica dell'amore: una fenomenologia che si svincoli da tutti i presupposti non avrebbe potuto distinguere nell'azione etica dualisticamente una realtà della vita e una realtà dello spirito. Questo dualismo lo porta a un dualismo fra eros platonico-greco come amore vitale e agàpe cristiana come amore personale-spirituale: da un lato, l'eros non basta per la fondazione dell'etica, ma neanche l'agàpe, se ridotto ad amore puramente spirituale, è sufficiente. Tuttavia, questa soluzione porta a considerare positivamente l'aspetto eudemonistico-egoistico dell'eros, non permettendo di comprendere anche l'amore fisico come forma di amore effettivo dell'altro (cioè come effettivo agàpe cristiano originario, che è amore totale fisico-vitale-spirituale).

In questo modo, Scheler non riesce a vedere nel cristianesimo un'etica cosmica, universale, onnicomprensiva e rivolta al rispetto di ogni vita e anche al rispetto della Natura: per Scheler, l'etica cristiana comporterebbe anzi una volontà di dominio della Natura e una prospettiva acosmica spirituale e quindi antropocentrica; e, laddove l'esempio di san Francesco d'Assisi, introdotto nella seconda edizione del 1923 di Essenza e forme della simpatia (Scheler [1913] 1923), attento a tutta la Natura e comprendente, in maniera non gerarchica, la fraternità e la sorellanza di tutte le creature, contraddice questa sua visione, si inventa che la posizione di san Francesco si può spiegare solo come caso di una fusione di eros greco e di agàpe cristiana. Scheler immagina così un'influenza dell'eros della poesia provenzale (a sua volta determinata, secondo Scheler, da influenze arabe) su Francesco; ma Scheler, anche ammessa questa influenza, non tiene in conto il fatto che quasi certamente la poesia provenzale si è formata a partire da influenze catare: l'eresia catara era diffusa nell'area provenzale. I catari, che derivavano la loro prospettiva cristiana dall'eresia manichea e che riprendevano il nome dall'eresia dei Katharoi del terzo secolo, erano vegetariani e predicavano proprio una uguaglianza di tutte le creature viventi di fronte a Dio. Che questa fosse proprio la prospettiva del cristianesimo originario e di Gesù è dimostrato da alcuni agrapha (Asin et Palacios 1917, 1926; Deleanu 2002, 25-35, 48) e, fra molte altre, dalla testimonianza di Eusebio nella Dimostrazione evangelica (Eusebio 2000, libro III, 74). Per negare questo respiro cosmico all'etica di Gesù - certamente presente come si vedrà più avanti - Scheler si improvvisa maldestramente esegeta del Nuovo Testamento, non tenendo conto dei testi originali o delle varianti.

La com-passione cosmica cristiana autentica si basa su un brano del Vangelo di Matteo in cui Gesù, identificato con il "Figlio dell'uomo", con il re-pastore e con il Signore, consapevolmente si auto-comprende 
come tutt'uno con tutte le creature che soffrono e identifica amore di Dio e amore di tutte le creature:

Matteo 25.31-46: ${ }^{31}$ Quando il Figlio dell'uomo verrà nella sua gloria con tutti i suoi angeli, si siederà sul trono della sua gloria. ${ }^{32} \mathrm{E}$ saranno riunite davanti a lui tutte le genti, ed egli separerà gli uni dagli altri, come il pastore separa le pecore dai capri, ${ }^{33} \mathrm{e}$ porrà le pecore alla sua destra e i capri alla sinistra. ${ }^{34}$ Allora il re dirà a quelli che stanno alla sua destra: Venite, benedetti del Padre mio, ricevete in eredità il regno preparato per voi fin dalla fondazione del mondo. ${ }^{35}$ Perché io ho avuto fame e mi avete dato da mangiare, ho avuto sete e mi avete dato da bere; ero forestiero e mi avete ospitato, ${ }^{36}$ nudo e mi avete vestito, malato e mi avete visitato, carcerato e siete venuti a trovarmi. ${ }^{37}$ Allora i giusti gli risponderanno: Signore, quando mai ti abbiamo veduto affamato e ti abbiamo dato da mangiare, assetato e ti abbiamo dato da bere? ${ }^{38}$ Quando ti abbiamo visto forestiero e ti abbiamo ospitato, o nudo e ti abbiamo vestito? ${ }^{39} \mathrm{E}$ quando ti abbiamo visto ammalato $\mathrm{o}$ in carcere e siamo venuti a visitarti? ${ }^{40}$ Rispondendo, il re dirà loro: In verità vi dico: ogni volta che avete fatto queste cose a uno solo di questi miei fratelli-sorelle creature più piccole, l'avete fatto a me. ${ }^{41} \mathrm{Poi}$ dirà a quelli alla sua sinistra: Via, lontano da me, voi artefici del male, nel fuoco dell'eone futuro, preparato dal diavolo per i suoi messaggeri. ${ }^{42}$ Perché ho avuto fame e non mi avete dato da mangiare; ho avuto sete e non mi avete dato da bere; ${ }^{43}$ ero forestiero e non mi avete ospitato, nudo e non mi avete vestito, malato e in carcere e non mi avete visitato. ${ }^{44}$ Anch'essi allora risponderanno: Signore, quando mai ti abbiamo visto affamato o assetato o forestiero o nudo o malato o in carcere e non ti abbiamo assistito? ${ }^{45} \mathrm{Ma}$ egli risponderà: In verità vi dico: ogni volta che non avete fatto queste cose a uno di questi miei fratelli-sorelle creature più piccole, non l'avete fatto a me. ${ }^{46} \mathrm{E}$ se ne andranno, questi nel dolore all'eone futuro, e i giusti alla vita futura eterna.

È stato Albert Schweitzer a mostrare per primo che il termine solitamente tradotto con "minimi fratelli" aveva una connotazione non meramente umana (Schweitzer [2001] 2002, 56), ma si riferiva a tutti i viventi e che quindi implicava in Gesù una consapevole forma di consapevole unipatia cosmo-vitale nella sofferenza: questo brano implica anche che non c'è differenza in Gesù fra amore di Dio e amore del prossimo, e smentisce su tutti i fronti l'interpretazione del cristianesimo di Scheler (san Francesco sicuramente si è ispirato a questo brano). Questa forma di unipatia presente in Gesù è mostrata anche da altre testimonianze. Negli Atti apocrifi di Giovanni 94-96, è riportato un inno di Gesù:

$\mathrm{Ci}$ ordinò di fare un cerchio tenendoci l'un l'altro per mano [...] Poi prese a cantare un inno, dicendo: "[...] Ti ringraziamo, Luce, nella quale non abita alcuna tenebra [...] La Grazia danza [...] danzate tutti. Amen [...] Il numero dodici delle stelle danza in alto. Amen. Il tutto partecipa alla

Relations - 9.1-2 - November 2021

https://www.ledonline.it/Relations/ - Online ISSN 2280-9643 - Print ISSN 2283-3196 
danza e colui che danza appartiene al tutto. Amen. Colui che non danza ignora ciò che è accaduto. Amen [...] Rispondi ora alla mia danza, vedi te stesso in me che parlo [...] Tu che danzi comprendi ciò che faccio, 96.32. giacché è anche tua questa sofferenza di tutti gli esseri innocenti che io sopporterò $[\ldots]$ Io sono la Parola che ha danzato con tutte le cose $[\ldots]$ ". (Moraldi 1971; corsivo aggiunto)

Nel Vangelo copto di Tommaso, logion 77, è detto da Gesù: "Io sono la luce che è su ogni cosa. Io sono il tutto: il tutto è uscito da me e il tutto a me è ritornato. Spaccate un legno, io sono là; alzate la pietra e là mi troverete". Similmente, nel papiro di Ossirinco1, Gesù dice: "Alza la pietra / e là mi troverai. / Spacca il legno / ed ivi io sono” (Moraldi 1971).

Che anche questi brani vadano interpretati non nei termini di una mera onnipresenza divina, ma piuttosto di una consapevole unipatia nella sofferenza e capacità di com-passione, è testimoniato dall'interpretazione cristiano-manichea di uno Iesus patibilis, in particolare di Fausto di Milevi: "Gesù, la Vita e la Salvezza degli uomini, è appeso a qualsiasi legno (patibilis Iesus, suspensus ex ligno)", come discusso polemicamente in Agostino, nelle Esposizioni sui Salmi (Agostino 1978-2009a, vol. XXVIII/2, 140, 12): [...] qui autem, inquiunt, agricola est, multum laedit crucem luminis. Quaeris quam crucem luminis? Membra, inquiunt, illa Dei quae capta sunt in illo proelio, mixta sunt universo mundo, et sunt in arboribus, in herbis, in pomis, in fructibus. Gesù (o Dio in Gesù) nella sua vita, e massimamente sulla croce, partecipa a tutto il dolore del mondo, non solo umano, ma di tutti gli esseri (Agostino 1978-2009b, vol. XIII/1-2, XIV).

In ogni caso, Scheler definisce la prospettiva di Francesco quella di un amore che costituisce una forma di consapevole spiritualizzata unipatia cosmo-vitale: l'unipatia (Einsfüblung) è considerata soprattutto una originaria e primitiva, inconscia, capacità di auto-identificazione dell'individuo con il resto dell'universo, di "sentire" all'unisono con l'universo. Mentre le religioni orientali arcaiche valorizzerebbero una forma di unipatia inconscia "negativa", basata sulla capacità di sentire come propria la sofferenza di ogni vivente e del mondo considerato vivente (la filosofia buddhista della com-passione ne sarebbe una razionalizzazione), differentemente la religione greca panica valorizzerebbe una forma di unipatia inconscia "positiva", basata sulla capacità di sentire lo stesso eros in tutta la Natura (la metafisica dell'eros di Platone e poi di Aristotele, fino al loro inglobamento in Agostino, in Tommaso e da questo in Dante che scrive l'amor che move il sole e l'altre stelle, non ne sarebbe che una razionalizzazione). Tuttavia, in questa distinzione, Scheler non sottolinea abbastanza che, mentre la prima forma orientale di unipatia si è tradotta

Relations - 9.1-2 - November 2021

https://www.ledonline.it/Relations/ - Online ISSN 2280-9643 - Print ISSN 2283-3196 
nell'affermazione consapevole di una solidale forma di co-sentire (Mitfüblen, sympathisieren), di com-passione, la seconda forma occidentale di unipatia effettivamente si è tradotta nell'affermazione consapevole non di un effettivo co-sentire, ma piuttosto di una meramente comune pulsione individualistica ed egoistica.

Certamente, la critica da parte di Friedrich Nietzsche (1844-1900) al sentimento della com-passione ha evidenziato che in alcune forme religiose, esemplarmente nel buddhismo, questa si riduce a un atteggiamento distaccato di superiorità e di "mera pietà" nei confronti di chi soffre, provato mentre si è assorti nella meditazione, e non è affatto un coinvolgimento nella sofferenza di un effettivo co-sentire e meno che mai un amore attivo nella cura delle sofferenze; tuttavia, Nietzsche non tiene conto che già in Arthur Schopenhauer (1788-1860) la com-passione ha inglobato in sé elementi cristiani di com-passione non riducibile a quella buddhista e che non possono essere oggetto della stessa critica (la confusione in Nietzsche ha la sua radice nell'interpretazione di Schopenhauer che mischia cristianesimo e buddhismo).

Scheler continua, però, in qualche modo e con un senso positivo, il tentativo di Nietzsche di mostrare come sia l'etica a determinare la metafisica: Nietzsche aveva mostrato come l'etica di Platone fosse alla base della sua metafisica del mondo delle idee per assicurare che la ricerca del bene avesse come esito la felicità individuale (eudemonia). Scheler mette così in evidenza che Schopenhauer ha dedotto dalla com-passione la sua metafisica monistica della volontà unica universale che soggiace alle apparenti volontà individuali, e, allo stesso modo, che Eduard von Hartmann (1842-1906) ha dedotto dall'amore la sua metafisica monistica. Ma la fenomenologia del sentire di Scheler mostra che solo considerando l'unipatia nella sua forma inconscia si sarebbe potuti arrivare a una metafisica monistica, mentre la com-passione e l'amore, che implicano un'unipatia consapevole dell'alterità e non una mera identificazione in cui l'io e il tu restano indistinti nell'inconscio, non possono definire una metafisica monistica, perché l'altro non può essere riassorbito nell'egoità e la com-passione e l'amore implicano l'irriducibilità dell'altro.

Scheler si chiede, allora, a quale comprensione nuova dell'essere delle cose ci apra l'amore come forma più alta di co-sentire; e la risposta è che il nostro essere in realtà non è un essere individuale ma è un conessere, un con-essere-l'uno-con-l'altro: la nostra identità non coincide con la nostra egoità, ma contiene in sé gli altri, anzi, nell'unipatia cosmovitale consapevole, comprende in sè tutto l'universo vivente; il noi, che si può estendere all'universo intero, precede l'io, e l'io nasce solo da una separazione indebita dagli altri.

Relations - 9.1-2 - November 2021

https://www.ledonline.it/Relations/ - Online ISSN 2280-9643 - Print ISSN 2283-3196 
Ralph Acampora (Acampora 2006) cerca di sviluppare una prospettiva simile, non tenendo conto di Scheler e della sua fondazione etica dell'ontologia, restando paradossalmente più vicino a una prospettiva ontologica heideggeriana, che aveva neutralizzato ontologicamente il con-essere scheleriano.

Scheler così fonda una nuova "ontologia etica". Questo con-essere è quindi più precisamente un con-amare, che costituisce la nostra "natura" più profonda che abbiamo tradito e continuamente tradiamo: l'essenza va compresa in termini temporali di originarietà da cui ci siamo allontanati; tutto è originariamente amore. Certamente questa prospettiva Scheler l'ha derivata dal cristianesimo: da una parte, Dio come agàpe è considerato come Trinità, cioè come tre persone ma una sola sostanza (essere); dall'altra, in san Paolo, ripreso anche da Agostino, si parla di un unico corpo mistico-cosmico di Cristo di cui gli individui sono membra, realizzata anche attraverso l'eucarestia che, come cibo non-violento, ci trasforma in unico corpo comunione d'amore. Il mistero della Trinità o del Corpo mistico di Cristo non sono considerati come meri dogmi, ma come aperture a una nuova comprensione della realtà. Secondo Scheler, è quindi la fenomenologia dell'Amore che ci rivela nel con-essere l'impronta del Dio-Amore cristiano: la prova dell'esistenza di Dio-Amore non ci può certo essere data da una dimostrazione razionale metafisica, ma dall'amore stesso.

Il nucleo del pensiero di Scheler è quindi etico, ha le sue radici nel cristianesimo e nel pensiero romantico tedesco dell'Ottocento: anche se li critica, Scheler riprende a suo modo Schopenhauer e Eduard von Hartmann (letteralmente "L'uomo-cuore"); mentre la fenomenologia ne costituisce il metodo.

Tuttavia, Scheler è all'origine anche della "fenomenologia ermeneutica" o dell' "ermeneutica fenomenologica", che poi sarà sviluppata da Martin Heidegger (1889-1976). Scheler abbandona la fenomenologia della percezione di Husserl e la sostituisce con una fenomenologia della comprensione (Scheler 1913, 1923), facendo una sintesi del pensiero di Husserl con quello di Wilhelm Dilthey (1833-1911). Per Scheler il nostro incontro originario con le cose e con il mondo non è percettivo di dati materiali: per noi le cose e il mondo si presentano primariamente non nella loro mera materialità, ma anche nella loro psichicità e nella loro spiritualità, nel loro senso e non loro "valore" etico: quando c'è, per esempio, un uomo con le mani giunte o rivolte verso il cielo, noi non vediamo soltanto il suo corpo materiale e poi da questo deduciamo logicamente che sta pregando, ma interpretiamo e comprendiamo direttamente la sua psichicità, la sua spiritualità, il senso e il valore del gesto. La compren- 
sione effettiva è una partecipazione etica del nostro essere all'essere delle cose e del mondo, non una spiegazione razionale che da percezioni materiali inferisce altre qualità.

Secondo Scheler, non è vero quanto affermato da Cartesio, e rielaborato da Husserl, che si abbia solo un'auto-percezione immediata dell'io dalla cui primaria certezza tutto il resto si deve inferire: possiamo "percepire-comprendere" gli altri nello stesso modo immediato dell'io, possiamo "percepirne" immediatamente i sentimenti e i pensieri come nostri, e la distinzione fra io e tu è meramente secondaria. Se per Fichte anche l'esistenza degli altri doveva essere dedotta da un postulato etico dell'io, per Scheler invece nella intuizione etica immediata dell'amore ci auto-comprendiamo primariamente come un noi cosmico al quale apparteniamo e di cui partecipiamo.

All'eudemonismo individualistico corrisponde un egoismo e un solipsismo metafisico, all'etica dell'amore corrisponde un con-essere universale in una "unipatia cosmico-vitale" dell'uomo anche "con le nuvole e col vento", con le piante, ma soprattutto con gli altri animali. Da qui, la possibilità di una nuova forma di etica del rispetto della vita, che, se integrata con una fenomenologia della vita vegetale e animale e delle loro differenze (le piante non soffrono per la raccolta del frutto, che costituisce piuttosto una forma di dono in cui si stabilisce una simbiosi evolutiva con gli animali che se ne nutrono), può declinarsi in una nuova forma di antispecismo vegano.

\section{RIFERIMENTI BIBLIOGRAFICI}

Acampora, Ralph R. 2006. Corporal Compassion: Animal Ethics and Philosopby of Body. Pittsburgh: University of Pittsburgh Press (trad. it. a cura di M. Maurizi e M. Filippi, Fenomenologia della compassione. Etica animale e filosofia del corpo, Sonda: Casale Monferrato, 2008).

Agostino, Aurelio. 1978-2009a. Esposizioni sui Salmi. In Opera omnia, edizione con testo latino a fronte, XXVIII/2. Roma: Città Nuova.

Agostino, Aurelio. 1978-2009b. Polemica con i Manichei. In Opera omnia, edizione con testo latino a fronte, XIII/1-2, XIV. Roma: Città Nuova.

Arendt, Hannah. 1929. Liebesbegriff bei Augustin. Versuch einer philosophischen Interpretation. Berlin: Julius (trad. it. a cura di L. Boella, Il concetto d'amore in Agostino. Saggio d'interpretazione filosofica, Milano: SE, 2001).

Asin et Miguel Palacios, edd. 1917, 1926, 1990, 2003. Logia et Agrapha Domini Jesu - Apud moslemicos scriptores, asceticos praesertim, usitata I et II. In $\mathrm{Pa}$ trologia Orientalis, t. 13, fasc. 3, n. 64 (1917) e t. 19, fasc. 4, n. 94 (1926). Turnhout (Belgium): Brepols.

Relations - 9.1-2 - November 2021

https://www.ledonline.it/Relations/ - Online ISSN 2280-9643 - Print ISSN 2283-3196 
Calogero, Guido. 1932. Etica. In Enciclopedia Treccani (1932), XIII, 447b-454a; poi come Schizzo di una storia dell'etica. In Saggi di Etica e di teoria del diritto, 106-139. Bari: Laterza, 1947.

Deleanu, Daniel, ed. 2002. The Ascetic Jesus in the Islamic Jesus: The Portrait of Jesus in Islamic Literature and Tradition, edited and translated from Arabic and Latin by D. Deleanu and J. Robson. Lincoln (NE): Writers Club Press.

Derrida, Jacques. 2006. L'animal que donc je suis, édité par M.-L. Mallet. Paris: Galilée (trad. it. di M. Zannini, a cura di G. Dalmasso, L'animale che dunque sono, Milano: Jaca Book, 2006).

Eusebio di Cesarea. 2000. Demonstratio Evangelica, trad. it. a cura di P. Carrara, Dimostrazione evangelica. Milano: Paoline.

Heidegger, Martin. 1927, 2001. Sein und Zeit. Tübingen: Niemeyer; poi in Gesamtausgabe, II, herausgegeben von F.-W. von Hermann. Frankfurt am Main: Klostermann, 1977 (la prima con le glosse a margine dell'Hüttenexemplar di Heidegger) (trad. it. di P. Chiodi, Essere e tempo, Longanesi: Milano, 1970, con aggiornamento bio-bibliografico di A. Marini, 1976; nuova edizione italiana a cura di F. Volpi sulla versione di P. Chiodi con le glosse a margine dell'Hüttenexemplar [Esemplare della baita] di Heidegger, Longanesi: Milano, 2005; trad. it., con testo tedesco a fronte, di A. Marini, Essere e tempo, Milano: Mondadori, 2006).

Moraldi, Luigi, a cura di. 1971, 1994. Apocrifi del Nuovo Testamento, I-III, prima edizione Torino: Utet; poi Casale Monferrato: Piemme.

Regan, Tom. 1983. The Case for Animal Rights. Berkeley: University of California Press (trad. it. di R. Rini, I diritti animali, Milano: Garzanti, 1990).

Regan, Tom, and Andrew Linzey. 1988. Animals and Christianity: A Book of Readings. New York: Crossroads (trad. it. Gli animali e il cristianesimo. Per un'etica compassionevole, Casale Monferrato: Sonda, 2012).

Regan, Tom, and Peter Singer. 1976. Animal Rights and Human Obligations. Englewood Cliffs (NJ): Prentice Hall (trad. it. di P. Garavelli, Diritti animali, obblighi umani, Torino: Edizioni Gruppo Abele, 1987).

Resta, Caterina. 2003. L'evento dell'altro. Etica e politica in Jacques Derrida. Torino: Bollati Boringhieri.

Scheler, Max. 1913, 1923. Wesen und Formen der Sympatbie. Bern: Cohen (trad. it. di L. Pusci, Essenza e forme della simpatia, Roma: Città Nuova, 1980; trad. it. di L. Oliva e S. Soannini, a cura di L. Boella, Milano: FrancoAngeli, 2010).

Scheler, Max. 1913-1916, 1921, 1927. Der Formalismus in der Ethik und die materiale Wertethik. Neuer Versuch der Grundlegung eines ethischen Personalismus, herausgegeben von M. Frings. Bonn: Bouvier, 2009 (trad. it., con testo tedesco a fronte, a cura di R. Guccinelli e con una presentazione di R. De Monticelli, Il formalismo nell'etica e l'etica materiale dei valori. Nuovo tentativo di fondazione di un personalismo etico, Milano: Bompiani, 2013).

Scheler, Max. 1914-1916, 1933. Ordo amoris. In Schriften aus dem Nachlass, I: Zur Ethik und Erkenntnislebre, 227-261. Berlin: Der Neue Geist (trad. it. a cura di E. Simonotti, Ordo amoris, Brescia: Morcelliana, 2008; trad. it., con testo tedesco a fronte, a cura di L. Iannascoli, Ordo amoris, Roma: Aracne, 2009).

Relations - 9.1-2 - November 2021

https://www.ledonline.it/Relations/ - Online ISSN 2280-9643 - Print ISSN 2283-3196 
Scheler, Max. 1915, 1963. Liebe und Erkenntnis. In M. Scheler, Gesammelte Werke I-XIII, VI: Schriften zur Soziologie und Weltanschauungslebre, herausgegeben von Maria Scheler, 77-98. München - Bern: Francke Verlag (trad. it. a cura di L. Pesante, Amore e conoscenza, Padova: Liviana, 1967; trad. it., con testo tedesco a fronte, a cura di L. Iannascoli, Liebe und Erkenntnis. Amore e conoscenza, Roma: Aracne, 2010).

Scheler, Max. 1917, 1921. Vom Wesen der Philosophie und der moralischen Bedingung des philosophischen Erkennens. In Vom Ewigen im Menschen. Leipzig: Reinhold - Verlag der Neue Geist; poi in Gesammelte Werke, V, herausgegeben von Maria Scheler, 61-99. Bern - München: Francke Verlag, 1954, 2000 (trad. it. di Ubaldo Pellegrino, L'essenza della filosofia, in L'eterno nell'uomo, Milano: Fabbri, 1972 e Roma: Edizioni Logos, 1991; L'essenza della filosofia, a cura di C. Amicantonio, Catanzaro: Rubettino, 2001; poi L'essenza della filosofia, in L'eterno nell'uomo, trad. it., con testo tedesco a fronte, a cura di P. Premoli De Marchi, 222-305, Milano: Bompiani, 2009).

Scheler, Max. 1928. Die Stellung des Menschen im Kosmos. Darmstadt: O. Reichl (trad. it. a cura di R. Padellaro, La posizione dell'uomo nel cosmo, in La posizione dell'uomo nel cosmo e altri saggi, Milano, Fabbri, 1970; poi a cura di M.T. Pansera, 115-191, Roma: Armando, 1997; trad. it. a cura di G. Cusinato dall'edizione originale del 1928, La posizione dell'uomo nel cosmo, Milano: FrancoAngeli, 2000, 2009).

Schweitzer, Albert. 2001. Predigten 1898-1948, herausgegeben von R. Brüllmann und E. Grässer. München: Beck (trad. it. parziale a cura di E. Colombo, Predica della Domenica 2 Marzo 1919, Servizio mattutino a St. Nicolai, in La melodia del rispetto per la vita. Prediche di Strasburgo, Milano: Paoline, 2002).

Singer, Peter. 1975, 1990. Animal Liberation. New York: Avon Books (trad. it. di P. Cavalieri e E. Ferreri, a cura di P. Cavalieri, Liberazione animale, Milano: Mondadori, 1991). 